\title{
Evaluación de conocimientos y prácticas sobre la leishmaniasis tegumentaria en un área endémica de Venezuela
}

\author{
Elsa Nieves¹, Néstor Villarreal'1, Maritza Rondón ${ }^{1}$, Mireya Sánchez², José Carrero³ \\ ${ }^{1}$ Laboratorio de Parasitología Experimental, Departamento de Biología, Facultad de Ciencias, Universidad \\ de Los Andes, La Hechicera, Mérida, Venezuela \\ ${ }^{2}$ Escuela Bolivariana Juan XXIII, Pan de Azúcar, Ejido, Mérida, Venezuela \\ ${ }^{3}$ Servicio de Dermatología de Tovar, Módulo Las Acacias, Tovar, Mérida, Venezuela
}

Introducción. La leishmaniasis es un grave problema de salud pública en muchas partes de América. Las poblaciones expuestas a la leishmaniasis carecen de información de la enfermedad, razón por la cual es necesario realizar intervenciones y evaluaciones educativas que contribuyan a que el control tenga un mayor impacto.

Objetivo. Determinar el nivel de conocimientos y prácticas sobre la leishmaniasis tegumentaria y la fauna de flebótomos en las comunidades endémicas de Bolero Alto y Bajo del municipio Pinto Salina del Estado Mérida, Venezuela, entre septiembre de 2006 y julio de 2007.

Materiales y métodos. Se elaboró una encuesta en la cual se incluyeron aspectos epidemiológicos, de prevención y de control de la leishmaniasis. Se aplicaron a personas mayores de siete años en viviendas seleccionadas aleatoriamente. También se realizaron capturas intradomiciliarias y peridomiciliarias de flebotominos vectores.

Resultados. Más del $68 \%$ de los individuos de las comunidades poseía un nivel de conocimientos considerado como insuficiente; los aspectos de mayor desconocimiento fueron en relación con la transmisión y la prevención. Se detectaron siete especies de Lutzomyia de importancia epidemiológica: L youngi, L. ovallesi, L. gomezi, L. walkeri, L. panamensis, L. punctigeniculata y $L$. venezuelensis. Las especies predominantes para ambas comunidades fueron L. youngi con más del $55 \%$ y L. ovallesi con más del $24 \%$ del total de especímenes capturados.

Conclusión. Se determinó un bajo nivel de conocimientos sobre la leishmaniasis en los pobladores de las comunidades endémicas estudiadas, lo cual se debe tener en cuenta en el desarrollo de alternativas educativas de impacto en el control complementario de la enfermedad. Las mismas deben dirigirse a cubrir las deficiencias de conocimientos más acentuadas en la población, enfocadas a evitar las picaduras del insecto vector.

Palabras claves: conocimientos, actitudes y práctica en salud, leishmaniasis/epidemiología, Psychodidae, vectores de enfermedades, Venezuela.

\section{Evaluation of knowledge and practice on tegumentary leishmaniasis in an endemic area of} Venezuela

Introduction. Leishmaniases constitutes a serious public health problem in many parts of the Americas. However, the populations exposed to leishmaniasis lack information about this disease. For this reason, educational assessments and interventions were deemed necessary to contribute to a greater impact of control measures.

Objective. The level of knowledge and practices was evaluated for tegumentary leishmaniasis and the phlebotomine sand fly vector species.

Materials and Methods. Between September 2006 and July 2007, a survey was conducted on epidemiological aspects, prevention, and control of leishmaniasis in two endemic communities-Bolero Alto and Bajo, in the municipality of Pinto Salina, Mérida state, Venezuela. It was administered to persons 7 years of age, in randomly selected houses. Collections of sand fly vectors were made indoors and around the houses.

Results. Approximately $68 \%$ of the population showed a level of knowledge on leishmaniasis considered as insufficient. The lowest level of knowledge found was on matters related to 
leishmaniasis transmission and prevention. Seven epidemiologically important Lutzomyia species were identified: L. youngi, L. ovallesi, L. gomezi, L. walkeri, L. panamensis, L. punctigeniculata and $L$. venezuelensis. The predominant species in both communities were $L$. youngi and L. ovallesi, constituting $55 \%$ and $24 \%$, respectively, of the totals.

Conclusions. The residents of the endemic communities studied had a low level of knowledge about leishmaniasis. This must be considered in the development of educational alternatives that complement control programs. A particular focus on the prevention of insect bites is recommended.

Key words: health knowledge, attitudes, practice; leishmaniasis/epidemiology, Psychodidae, disease vectors, Venezuela.

La leishmaniasis es causada por un protozoario del género Leishmania. Este parásito se transmite a los seres humanos mediante la picadura de flebotominos vectores, previamente infectados por medio de la ingestión sanguínea sobre un huésped vertebrado infectado (1-3). El control de la leishmaniasis se basa, fundamentalmente, en la detección de casos activos y su tratamiento.

En la actualidad, no existe ningún modelo bien definido para un control costo eficaz $(4,5)$. La lucha antivectorial mediante fumigaciones es imposible en la mayoría de los casos, debido al desastre ecológico que genera $(4,6)$. Aunque una vacuna podría constituir una medida preventiva real, su desarrollo aún está en fase de prueba y, además, falta conocer los diversos matices inmunológicos en la interacción huésped-parásito (7). El conocimiento de las especies vectoras del género Lutzomyia y de su comportamiento resulta fundamental para proponer medidas eficaces de control.

Por otro lado, muchos pacientes no son concientes de los síntomas iniciales de la enfermedad y en la mayoría de los casos las comunidades carecen de sistemas de salud (3,7-9). En las campañas de control no se consideran las costumbres de los pobladores en las comunidades endémicas, por lo que es necesario realizar intervenciones y evaluaciones educativas que contribuyan a que el control tenga un mayor impacto $(10,11)$. Los

\footnotetext{
Correspondencia:

Elsa Nieves, Laboratorio de Parasitología Experimental, Departamento de Biología, Facultad de Ciencias, Universidad de Los Andes, La Hechicera, Mérida, Estado Mérida, 5101, Venezuela.

Teléfono: (+582) 74240 1244; fax: (+582) 2742401286

nevelsa@ula.ve
}

Recibido: 09/10/07; aceptado:02/05/08 estudios de diferentes partes del mundo han demostrado que las poblaciones expuestas carecen de información sobre la enfermedad, como lo reportan varios autores en Brasil $(12,13)$ y en Colombia $(14,15)$.

En los últimos años ha ocurrido un aumento de la leishmaniasis debido a los cambios climáticos, naturales y provocados por la población, que incrementan esta enfermedad zoonótica, aunado al hecho de que el avance en los métodos de diagnóstico facilita su detección $(16,17)$. La leishmaniasis es endémica en 88 países del mundo y se considera que 350 millones de personas corren el riesgo de contraer esta enfermedad $(7,18)$.

En Venezuela, la leishmaniasis presenta una amplia distribución geográfica, con predominio en las regiones montañosas, de piedemonte o selváticas, donde puede tener carácter endemoepidémico. Las verdaderas incidencia y prevalencia de la enfermedad se desconocen debido, entre otros factores, al subregistro de pacientes, a la deficiente recolección de datos, a la dificultad en el reconocimiento de las formas incipientes y de los casos con manifestaciones no clásicas (19-21).

Se han registrado casos en 23 de las 24 entidades federales, y la región de los Andes venezolanos es la que presenta mayor incidencia de leishmaniasis cutánea y mucocutánea (22). En el decenio 1996-2006 se reportó un promedio de 400 casos anuales en el estado Mérida (comunicación personal, J. Carrero).

La importancia de la leishmaniasis tegumentaria radica en su alta incidencia en las zonas rurales y periurbanas con alto impacto social que impiden el desenvolvimiento normal del trabajo y de las 
actividades cotidianas (21). Es necesario un manejo oportuno y adecuado de dicha parasitosis que permita establecer pautas de prevención y control con la participación activa de los afectados, de manera que se pueda concebir una política de capacitación de la población encaminada a la prevención de esta enfermedad.

En el presente estudio se evaluó el nivel de conocimientos de los pobladores de un área endémica de leishmaniasis en Venezuela y se determinó la fauna de flebótomos.

\section{Materiales y métodos}

\section{Área de estudio}

Se estudiaron dos comunidades mestizas rurales, ubicadas en la cordillera de los Andes venezolanos, las cuales presentan un relieve predominantemente montañoso de carácter asimétrico, separadas por fuertes pendientes, entre una superficie irregular con poca cubierta vegetal y precipitaciones de $1.100 \mathrm{~mm}$. La zona corresponde a un bosque húmedo, siempre verde y con temperaturas promedios de $24^{\circ} \mathrm{C}$.

La comunidad de Bolero Bajo (8॰30'22' N$71^{\circ} 36^{\prime} 25^{\prime \prime} \mathrm{W}$ ) está situada a una altura de 738 msnm con 45 viviendas continuas y una población estimada de 250 habitantes, con mayor intervención de la vegetación primaria que la rodea, y la comunidad de Bolero Alto $\left(8^{\circ} 29^{\prime} 23^{\prime \prime} \mathrm{N}\right.$ $\left.71^{\circ} 36^{\prime} 25^{\prime \prime} \mathrm{W}\right)$ a $915 \mathrm{msnm}$ con 86 viviendas dispersas y una población estimada de 400 habitantes, con menor impacto de la vegetación primaria que la cubre. Ambas comunidades pertenecen a la parroquia de Mesa Bolívar del municipio Pinto Salina del estado Mérida, Venezuela, a $64 \mathrm{~km}$ de la capital del estado, separadas entre sí por una distancia de $6 \mathrm{~km}$.

En estas áreas, la leishmaniasis tegumentaria es tradicionalmente considerada como endémica. Las comunidades se caracterizan por poseer casas distribuidas a lo largo de zonas arborizadas, con plantaciones de café, cacao, platanares y árboles frutales. Rodeadas por montañas con cubiertas de vegetación primaria con poca intervención humana, su actividad económica está fundamentada en la explotación agrícola en la producción de café mediante el método tradicional de sombreo y de cacao.

\section{Diseño metodológico}

Los indicadores asociados con el nivel de conocimientos sobre la leishmaniasis y las medidas de protección se evaluaron mediante una encuesta. El cuestionario incluyó una serie de preguntas, tanto abiertas, como cerradas. Las últimas demandaron respuestas afirmativas 0 negativas que le restaron subjetividad a los resultados e hicieron que la información fuera más confiable (23). El cuestionario estaba conformado por preguntas sobre datos relacionados con la identificación personal, las condiciones de morada, los factores epidemiológicos relacionados con la transmisión, la presencia de animales domésticos, los cultivos principales, el insecto vector y el conocimiento de algunos aspectos de la enfermedad: preventivos, clínicos, terapéuticos y de transmisión, para un total de 40 preguntas.

Se encuestaron todas las personas mayores de siete años presentes en la vivienda en el momento de visitarla. Las casas encuestadas se seleccionaron al azar, mediante un muestreo sistemático de una casa por cada tres o cuatro viviendas. Las entrevistas se realizaron con muestrarios de insectos vivos de triatominos ("chipos") y flebotominos ("angoletas"), además de fotografías de lesiones cutáneas.

El tamaño de la muestra para la toma de la encuesta se determinó sobre el estimativo de la población total de residentes de cada comunidad según el censo nacional, para un nivel de confianza de 95\%. Los cálculos se hicieron con una calculadora digital (http://www.surveysystem. com/sscalce.htm), y se estimó un mínimo del tamaño para muestra en Bolero Bajo de 70 encuestas y para Bolero Alto de 64 encuestas.

El trabajo de campo en las comunidades lo llevó acabo un equipo de especialistas en el área de Parasitología de la Universidad de Los Andes y profesionales de la salud del Servicio de Dermatología de Tovar, Mérida, todos debidamente entrenados para la utilización de la planilla en el levantamiento de la base de datos.

Para determinar el nivel de conocimientos de los pobladores, se seleccionaron 20 preguntas del cuestionario que medían el conocimiento sobre 
la leishmaniasis: la enfermedad, el vector, la transmisión y la prevención; a cada pregunta se le asignó un punto. Los diseños para medir el nivel de conocimientos sobre la leishmaniasis y sobre la transmisión tenían igual proporción de preguntas. Según la puntuación obtenida en el nivel de conocimientos, se clasificó en dos categorías: insuficiente ( 0 a 10 puntos) y básico ( 11 a 20 puntos).

\section{Muestreo de flebotominos}

Para determinar la presencia del insecto vector, se realizó una captura mensual de flebotominos en el peridomicilio de ambas comunidades, utilizando trampas Shannon, colocadas en los alrededores de la vivienda a una distancia de 10 a $20 \mathrm{~m}$, en horario nocturno, entre las 19:00 y las 20:00 horas, y capturas intradomiciliarias utilizando trampas de papel aceitado de $20 \mathrm{~cm} x$ $29 \mathrm{~cm}$, durante 24 horas, colocando tres trampas por casa, ubicadas en la sala, el cuarto y la cocina.

Se muestreó el $10 \%$ de las viviendas de cada comunidad. Los ejemplares se trasladaron al laboratorio para su identificación taxonómica, utilizando las claves de Young y Duncan (24). Los ejemplares capturados vivos se disecaron bajo microscopio estereoscópico y se observaron bajo microscopio óptico para su identificación taxonómica y la determinación de los índices de infección natural.

\section{Análisis estadístico}

La información se ingresó a una base de datos utilizando el programa Epilnfo, versión 6. Se elaboraron tablas de distribución de frecuencia y tablas de contingencia de doble entrada. Para el análisis se aplicó la prueba de ji al cuadrado, con un nivel de significancia de $p<0,05$.

\section{Aspectos éticos}

Para la aplicación de las encuestas se obtuvo previamente el aval del Comité de Bioética de la Facultad de Ciencias de la Universidad de Los Andes, así como el consentimiento verbal de los entrevistados.

\section{Resultados}

Se encuestaron 143 pobladores en las dos comunidades estudiadas; $52 \%(n=75)$ provenía de la comunidad de Bolero Bajo y $48 \%(n=68)$ de Bolero Alto. El $40 \%$ de los pobladores encuestados en ambas comunidades estaba constituido por jóvenes entre 7 y 20 años, cerca del $36 \%$ por mayores de 36 años y cerca del $25 \%$ representado por individuos entre 21y 35 años. La población del sexo femenino predominó en ambas comunidades con valores cercanos a $56 \%$ (figura 1 ).

De la población encuestada, $15 \%$ de Bolero Alto y $4 \%$ de Bolero Bajo refirieron la presencia de cicatrices compatibles con leishmaniasis, mientras que $21 \%$ de los encuestados en Bolero Alto y $34 \%$ en Bolero Bajo manifestaron tener un familiar que había padecido la enfermedad y $51 \%$ de Bolero Alto y $45 \%$ de Bolero Bajo reconoció que un vecino había sufrido la enfermedad.

En relación con el nivel de conocimientos de la población sobre la leishmaniasis, un alto porcentaje manifestó haber escuchado hablar de dicha enfermedad, $82 \%$ en Bolero Alto y $80 \%$ en Bolero Bajo; $58 \%$ en Bolero Alto y $62 \%$ en Bolero Bajo reconocieron que la leishmaniasis era una enfermedad de la piel, y $68 \%$ en Bolero Alto y $47 \%$ en Bolero Bajo acertó en que la curación era con tratamiento médico (cuadro 1).

Por otro lado, gran parte de la población reconoció saber cómo se transmite la enfermedad; cerca

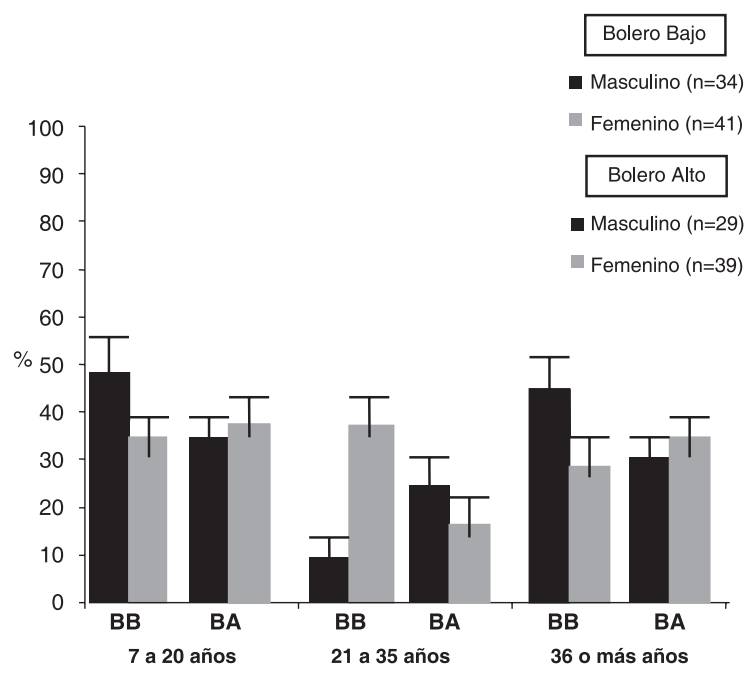

Figura 1. Sexo y grupos de edad de las personas encuestadas en las comunidades de Bolero Alto (BA) y Bolero Bajo (BB); barra: error estándar. 
Cuadro 1. Conocimientos y prácticas sobre leishmaniasis de los habitantes de las dos localidades estudiadas.

\begin{tabular}{|c|c|c|c|c|c|}
\hline Tema & Variable & $\begin{array}{l}\text { Bolero Alto \% } \\
\text { (personas/total) }\end{array}$ & $\begin{array}{l}\text { Bolero Bajo \% } \\
\text { (personas/total) }\end{array}$ & $\mathrm{Ji}^{2}$ & $p$ \\
\hline \multirow[t]{2}{*}{$\begin{array}{l}\text { Conocimiento de } \\
\text { la leishmaniasis }\end{array}$} & $\begin{array}{l}\text { Ha escuchado hablar } \\
\text { de la leishmaniasis }\end{array}$ & $82(56 / 68)$ & $80(60 / 75)$ & 0,13 & 0,720 \\
\hline & $\begin{array}{l}\text { Presencia de cicatrices } \\
\text { Familiar que haya padecido la enfermedad } \\
\text { De parte de un vecino haber padecido } \\
\text { la enfermedad } \\
\text { La leishmaniasis en una enfermedad } \\
\text { de la piel } \\
\text { La cura es con tratamiento }\end{array}$ & $\begin{array}{l}15(10 / 68) \\
21(14 / 68) \\
51(35 / 68) \\
58(39 / 68) \\
68(46 / 68)\end{array}$ & $\begin{array}{c}4(3 / 75) \\
34(26 / 75) \\
45(34 / 75) \\
\\
62(47 / 75) \\
47(35 / 75)\end{array}$ & $\begin{array}{l}4,91 \\
3,48 \\
0,53 \\
0,42 \\
6,35\end{array}$ & $\begin{array}{c}{ }^{*} 0,0267 \\
0,062 \\
0,464 \\
0,518 \\
{ }^{\star} 0,0117\end{array}$ \\
\hline $\begin{array}{l}\text { Conocimiento del } \\
\text { vector }\end{array}$ & $\begin{array}{l}\text { Saber que se transmite por picadura } \\
\text { de insecto } \\
\text { Admitieron al flebotomino como } \\
\text { transmisor } \\
\text { Reconoció que el transmisor era } \\
\text { la angoleta } \\
\text { Reconoció a los flebotominos vivos } \\
\text { como angoleta } \\
\text { Insectos vectores en el peridomicilio }\end{array}$ & $\begin{array}{l}70(48 / 68) \\
60(41 / 68) \\
14(10 / 68) \\
43(29 / 68) \\
100(68 / 68)\end{array}$ & $\begin{array}{c}71(53 / 75) \\
53(40 / 75) \\
19(14 / 75) \\
30(23 / 75) \\
100(75 / 75)\end{array}$ & $\begin{array}{l}0,03 \\
0,70 \\
0,40 \\
2,20 \\
0\end{array}$ & $\begin{array}{c}0,862 \\
0,403 \\
0,52 \\
0,138 \\
0\end{array}$ \\
\hline $\begin{array}{l}\text { Conocimiento del } \\
\text { ciclo de transmisión }\end{array}$ & $\begin{array}{l}\text { No relacionó la enfermedad con los } \\
\text { reservorios silvestres o domésticos } \\
\text { Relacionó la enfermedad con la } \\
\text { vegetación } \\
\text { Plantaciones de café en el peridomicilio } \\
\text { Tiene animales domésticos } \\
\text { Gallinero en el peridomicilio } \\
\text { Tener el baño fuera de la casa }\end{array}$ & $\begin{array}{l}57(39 / 68) \\
48(33 / 68) \\
93(63 / 68) \\
78(53 / 68) \\
49(23 / 68) \\
52(35 / 68)\end{array}$ & $\begin{array}{c}72(54 / 75) \\
14(11 / 75) \\
90(68 / 75) \\
100(75 / 75) \\
48(36 / 75) \\
40(30 / 75)\end{array}$ & $\begin{array}{r}19,06 \\
0,18 \\
18,35 \\
2,94 \\
1,88\end{array}$ & $\begin{array}{c}{ }^{*} 6,75 \times 10^{-2} \\
{ }^{*} 1,26 \times 10^{-5} \\
0,67 \\
{ }^{*} 1,83 \times 10^{-5} \\
{ }^{*} 8,66 \times 10-2 \\
0,170\end{array}$ \\
\hline $\begin{array}{l}\text { Uso de las medidas } \\
\text { de protección }\end{array}$ & $\begin{array}{l}\text { Usa mosquitero } \\
\text { Usa insecticida }\end{array}$ & $\begin{array}{c}10(7 / 68) \\
36(24 / 68)\end{array}$ & $\begin{array}{c}0(0 / 75) \\
46(35 / 75)\end{array}$ & $\begin{array}{l}8,06 * \\
1,89\end{array}$ & $\begin{array}{c}* 4,52 \times 10^{-3} \mathrm{de} \\
0,169\end{array}$ \\
\hline
\end{tabular}

* Estadísticamente significativo $p \leq 0,05$

Total: total de personas encuestadas

del $70 \%$ reconoció que la enfermedad se transmite por picadura de insecto en ambas comunidades y $60 \%$ en Bolero Alto y $53 \%$ en Bolero Bajo admitieron al flebotomino como transmisor. Sin embargo, en ambas comunidades menos del $20 \%$ respondió que el transmisor era la "angoleta" y sólo el $43 \%$ en Bolero Alto y $30 \%$ en Bolero Bajo reconocieron a los flebotominos vivos como la "angoleta".

En relación con el papel de los animales, $57 \%$ de los encuestados en Bolero Alto y $72 \%$ en Bolero Bajo no relacionaron la enfermedad con los reservorios silvestres o domésticos; $78 \%$ en Bolero Alto y $100 \%$ en Bolero Bajo tenían animales en el domicilio. En relación con la tenencia de animales domésticos en las dos comunidades, el
$100 \%$ de las casas de Bolero Bajo tenía cría de animales domésticos y el $55 \%$ en Bolero Alto, con predominio de la presencia de perros y gallinas. En ambas comunidades cerca del $49 \%$ poseían gallinero en el peridomicilio. Se determinó en orden creciente la presencia de los siguientes animales: burro, ardilla, vaca, oveja, pato, paloma, pavo, gato, conejo, cerdo, gallina y perro, con valores entre $4 \%$ y $80 \%$.

En relación con las medidas de protección, apenas el $10 \%$ en Bolero Alto y $0 \%$ en Bolero Bajo admitieron usar mosquitero. Menos de la mitad de la población usa insecticida, 36\% en Bolero Alto y $46 \%$ en Bolero Bajo. En cuanto a los factores ambientales, $93 \%$ en Bolero Alto y $90 \%$ en Bolero Bajo tienen cultivos de café en el 
peridomicilio, mientras que $48 \%$ en Bolero Alto y $14 \%$ en Bolero Bajo relacionaron la enfermedad con la vegetación silvestre.

La principal actividad nocturna de los pobladores era ver televisión, registrada en más del $60 \%$ de los encuestados en ambas comunidades.

Más del $60 \%$ de los grupos familiares estaban constituidos en promedio por más de cinco miembros por vivienda. El $52 \%$ en Bolero Alto y $40 \%$ en Bolero Bajo manifestaron tener el baño fuera de la casa.

Por otro lado, según la valoración realizada acerca del nivel de conocimientos sobre la leishmaniasis en las poblaciones encuestadas, se determinó que $68 \%$ de los individuos en Bolero Alto y $75 \%$ en Bolero Bajo poseían un nivel de conocimientos considerado como insuficiente. Las deficiencias más acentuadas fueron sobre el conocimiento del insecto vector, sobre la transmisión y sobre la prevención de la leishmaniasis, y estas diferencias fueron estadísticamente significativas $(p<0,05)$.

En relación con la presencia de los flebotominos vectores, se detectó sólo la presencia intradomiciliaria de una hembra de L. gomezi en la comunidad de Bolero Bajo. En cuanto a la biodiversidad de Lutzomyia en el peridomicilio, en el cuadro 2 se resumen las especies de flebotominos capturadas en las dos comunidades estudiadas. Se identificaron 7 especies de Lutzomyia de importancia epidemiológica en Bolero Alto, L. youngi (Feliciangeli \& Murillo, 1987); L. ovallesi (Ortiz, 1952); L. gomezi
(Nitzulescu, 1931); L. walkeri (Newstead, 1914); L. panamensis (Shannon, 1926); L. punctigeniculata (Floch \& Abonnenc, 1944) y L. venezuelensis (Floch \& Abonnenc, 1948), mientras que estas dos últimas especies no se encontraron en la comunidad de Bolero Bajo. Las especies predominantes en ambas comunidades fueron $L$. youngi con más del $55 \%$ y L. ovallesi con más del $23 \%$. No se detectó infección natural en 341 hembras disecadas, provenientes de las dos comunidades.

\section{Discusión}

La falta de información sobre la leishmaniasis en los afectados conlleva a la demora en la búsqueda de atención médica y, muchas veces, ocasiona complicaciones en el desarrollo de la enfermedad $(9,25)$. A pesar de la importancia de la leishmaniasis como problema de salud pública, son pocos los estudios de evaluación o intervenciones sobre el nivel de conocimiento en la población venezolana, que resalten la necesidad de la educación en las comunidades en zonas de alto riesgo. El sistema de encuesta diseñado en este trabajo permitió conocer la aptitud y la actitud de los pobladores de las comunidades estudiadas ante la leishmaniasis.

A pesar de que las comunidades estudiadas están localizadas en zonas endémicas, el conocimiento muchas veces se restringió a los pobladores que habían padecido la enfermedad o a aquéllos que presentaron casos en la familia o en un vecino. Situación semejante ha sido reportada por otros

Cuadro 2. Abundancia relativa de la fauna de flebótomos de las comunidades de Bolero Alto y Bolero Bajo con trampa Shannon (19:00 a 20:00 horas) en el peridomicilio*.

\begin{tabular}{|c|c|c|}
\hline \multirow[t]{2}{*}{ EspecieVlocalidad } & Bolero Alto $(\mathrm{Tc}=171)$ & Bolero Bajo $(\mathrm{Tc}=170)$ \\
\hline & \multicolumn{2}{|c|}{$\%$ (\# ejemplares) } \\
\hline L. youngi & $63,2(108)$ & $55,9(95)$ \\
\hline L. ovallesi & $32,7 \quad(56)$ & $23,5(40)$ \\
\hline L. gomezi & $1,2 \quad(2)$ & $10,6(18)$ \\
\hline L. walkeri & $1,2 \quad$ (2) & 8,8 (15) \\
\hline L. panamensis & 0,6 & $1,2 \quad$ (2) \\
\hline L. punctigeniculatus & $0,6 \quad(1)$ & $0,0 \quad(0)$ \\
\hline L. venezuelensis & $0,6 \quad(1)$ & $0,0 \quad(0)$ \\
\hline
\end{tabular}

Tc: total capturado de flebotominos

* $\mathrm{Ji}^{2}=28,57 ; \mathrm{p}=0,0000733$; hay diferencias significativas entre la fauna de flebótomos de las dos localidades $(\mathrm{p}<0,05)$. 
autores en Colombia, Brasil, Ecuador y Costa Rica $(9,13,25-27)$.

Se detectó un bajo nivel del conocimiento global sobre la leishmaniasis en ambas comunidades estudiadas; los aspectos de mayor desconocimiento fueron sobre la transmisión y la prevención. En Colombia, Isaza et al. (27) reportaron, en una zona de alta prevalencia, que $94 \%$ de la población estudiada reconocía a la leishmaniasis tegumentaria como una enfermedad de la piel.

En cuanto la transmisión, 35\% relacionó la enfermedad con picaduras de insecto. Alves et al. (8) en Brasil, en un estudio sobre el conocimiento de la población sobre leishmaniasis, reportó que $77,8 \%$ de los entrevistados desconocían las medidas de control, concluyendo que el conocimiento de la prevención y el tratamiento era bajo.

Otro factor observado en ambas comunidades estudiadas fue el hacinamiento, señalado como factor importante por considerar en la transmisión de la leishmaniasis (28). Además, se detectó en los pobladores el uso de poca ropa, que concurre para que una mayor área corporal esté expuesta al ataque de los insectos.

Según Desjeux (29), los principales factores de transmisión de la leishmaniasis tegumentaria que se debe considerar en diversas partes del mundo están relacionados con la urbanización, la deforestación, la presencia de animales domésticos y el desarrollo de la agricultura. La transmisión peridomiciliaria ocurre, principalmente, por la adaptación de los insectos vectores a los ambientes naturales modificados. En un estudio sobre leishmaniasis cutánea en Costa Rica, los autores reportaron que la transmisión ocurría tanto en el interior del domicilio como en el peridomicilio, y detectaron alta incidencia de la enfermedad en los niños y en las mujeres, y sugirieron que la prevención debe basarse en medidas caseras (25).

De acuerdo con los hallazgos del presente trabajo, probablemente la transmisión se efectuó, principalmente, en las áreas peridomiciliarias, en donde se detectó la presencia de varias especies de flebotominos incriminadas como vectores. Ello, tal vez, por ser comunidades con abundantes áreas silvestres a su alrededor, lo que indica que no ha ocurrido una adaptabilidad de los vectores a invadir las áreas intradomiciliarias y, probablemente, por la ausencia de reservorios secundarios domésticos que faciliten la transmisión intradomiciliaria (30).

A pesar de que las dos comunidades están relativamente cercanas, se encuentran separadas por una barrera geográfica que explica las diferencias encontradas. Además, la comunidad de Bolero Bajo fue la que presentó mayor impacto ambiental. En la comunidad de Bolero Bajo se registró menor número de especies de flebotominos en el peridomicilio y fue en ella en donde se detectó una hembra de L. gomezi intradomiciliaria.

Estas pequeñas diferencias bio-ecológicas entre las comunidades podrían ser fundamentales para determinar la especie de Lutzomyia transmisora. No se detectó infección natural en los flebotominos capturados en las dos comunidades estudiadas. En Venezuela existe poca información sobre la estacionalidad en la transmisión de la leishmaniasis tegumentaria. Apenas sabemos de su hipoendemicidad en casi todo el territorio venezolano y de la presencia de brotes cada cierto tiempo, muchas veces generados por actividades de quemas o deforestación en esas zonas, como también se reportan en otros países (20,31-33).

Por otro lado, es poco frecuente el hábito en los pobladores de las comunidades de fumigar las casas con insecticidas, posiblemente como consecuencia del bajo ingreso económico. La quema de plantas o basura podría constituirse en una medida de protección importante contra la leishmaniasis; costumbres como ésta deberían incentivarse como medidas alternativas de control comunitario $(12,27)$.

Una posible alternativa de prevención es evitar las picaduras de los insectos vectores. La interrupción del ciclo de Leishmania por medio del control del vector es una alternativa que permite ofrecer medidas complementarias de impacto al diagnóstico y tratamiento de casos $(34,35)$.

Como lo reseñan otros autores, los principales factores que favorecen el mantenimiento y el acercamiento de los insectos vectores al domicilio son la cría de animales domésticos, principalmente 
de gallinas, en la proximidad de las casas, junto con la tenencia de perros, así como la llegada de reservorios silvestres infectados a las áreas peridomiciliarias, los cuales a su vez infectan a la población de flebotominos que se refugia en ellas $(31,36)$.

Otro factor que favorece la presencia peridomiciliaria de los flebotominos son los cultivos de café $(28,37)$. Si bien no existe evidencia consolidada del cafetal como un sitio de refugio de los flebotominos, se han criado con éxito especies de Lutzomyia en el laboratorio con alimento a base de hojas de café $(38,39)$. Además, varias especies de Lutzomyia se han capturado en plantaciones de café (40) y hay evidencias de transmisión de Leishmania en plantaciones de café en Brasil (37).

La mayoría de las especies de flebotominos encontradas en las dos comunidades estudiadas son antropofílicas y se han incriminado como transmisoras de Leishmania. Los resultados no sólo reflejan la biodiversidad de especies de Lutzomyia en estas zonas endémicas, sino también resaltan la presencia de $L$. youngi con más del $55 \%$ y L. ovallesicon más del $23 \%$ como posibles vectores de especies de Leishmania circulantes en el peridomicilio en los Andes venezolanos. Resultados semejantes fueron reportados en la región de Las Maticas del estado Lara, Venezuela, a $1.360 \mathrm{msnm}$, en la cual tanto en el periodo de lluvia como en el de sequía, $L$. youngi $(96,54 \%)$ y $L$. ovallesi $(2,9 \%)$ fueron las especies más abundantes y consideradas como las principales transmisoras en la zona (41). $L$. ovallesiaparece entre los 300 y los 1.000 msnm, mientras $L$. youngi está descrita con preferencia por encima de los $800 \mathrm{msnm}$ de altitud. $L$ youngi es una especie más estricta en su condición estenotérmica que L. ovallesi. Sin embargo, ambas especies se encuentran en vegetación de bosque húmedo tropical (20).

La transmisión de leishmaniasis tegumentaria ocurre en una variedad de ambientes ecológicos; ambas especies se han capturado en cafetales. Estas especies, que también han sido incriminadas como transmisoras en el domicilio y en el peridomicilio en otros países (28,32,41-45), son originalmente zoofílicas y selváticas. Como resultado de la deforestación e intervenciones del ambiente, se han adaptado al peridomicilio y a alimentarse sobre humanos y animales domésticos, como ha ocurrido con otras especies peridomiciliarias (33).

Otros autores también proponen la participación comunitaria como parte complementaria en el control en las zonas endémicas, orientado al vector y a los reservorios (12), y algunos autores resaltan la importancia de concientizar a la población escolar, como lo propusieron Costa et al. (46).

Como en otros países de América, la prevención de la transmisión peridomiciliaria en el área rural constituye un problema de difícil resolución por la naturaleza y magnitud del problema $(13,42)$. La educación es una gran herramienta preventiva que debe ir orientada al uso de mosquiteros de malla fina, principalmente en los niños lactantes, uso de ventiladores, uso regular de insecticida residual domiciliario, uso de ropa como medida protectora, quema de hojas frecuentemente en el peridomicilio, mantener a los animales domésticos fuera del domicilio y uso de repelente artesanal durante las labores de campo $(43,44)$. El uso de estas medidas se ve limitado dependiendo del nivel de ingreso de la familia, como lo demuestran Santos et al, (13), en un área endémica del sur de Bahía, Brasil.

En conclusión, la metodología utilizada proporciona una herramienta de evaluación para mejorar la aplicación de medidas preventivas complementarias, orientadas a la educación en la población afectada. Las medidas que estimulen el evitar la picadura del insecto y el uso de repelentes caseros en situaciones de alto riesgo, pudieran ser medidas más eficientes, de bajo costo y menor impacto ambiental, que las intervenciones con insecticidas químicos. Es necesario, además, reformular las estrategias de control, que deben incluir nuevas políticas integrales de promoción de calidad de vida en las poblaciones endémicas de leishmaniasis con nuevos enfoques metodológicos y con mayor participación comunitaria preventiva.

\section{Agradecimientos}

A Irlanda Márquez; a cada uno de los pobladores de Bolero Bajo y Bolero Alto. 


\section{Conflicto de intereses}

Los autores declaramos que no existe conflicto de interés.

\section{Financiación}

Este trabajo fue financiado por FUNDACITEMérida, por CDCHT-ULA, Proyecto C-1406-06-03$B$ y Proyecto LOCTI.

\section{Referencias}

1. Grimaldi G Jr, Tesh RB. Leishmaniasis of the New World: current concepts and implications for future research. Clin Microbiol Rev. 1993;6:230-50.

2. Sánchez L, Sáens E, Pancorbo J, Zegarra R, Garcés $\mathbf{N}$, Regis A. Leishmaniasis. Dermatología Peruana. 2004;14:82-98.

3. Alvar J, Yactayo S, Bern C. Leishmaniasis and poverty. Trends Parasitol. 2006;22:552-7.

4. Alexander B, Maroli M. Control of phlebotomine sandflies. Med Vet Entomol. 2003;17:1-18.

5. Maroli M, Khoury C. Prevention and control of leishmaniasis vector: current approaches. Parassitologia. 2004;46:211-5.

6. Lainson R, Shaw JJ. Epidemiology and ecology of leishmaniasis in Latin-America. Nature. 1978;273:595-600.

7. OMS. Serie de Informes Técnicos No. 793. Ginebra: Organización Mundial de la Salud; 2006. p. 1-7.

8. Gama ME, Barbosa JS, Pires B, Cunha AK, Freitas AR, Ribeiro IR, et al. Avaliaçao do nivel de conhecimento de pupolações residentes em áreas endêmicas têm sobre leishmaniose visceral; Estado do Maranhâo, Brazil. Cad Saude Publica. 1998;14:381-90.

9. Uchoa CM, Serra CM, Magalhaes C de M, Silva RM, Figlioulo LP, Leal CA, et al. Health education: teaching about American tegumentary leishmaniasis. Cad Saude Publica. 2004;20:935-41.

10. Saravia NG, Nicholls RS. Leishmaniasis: un reto para la salud pública que exige concertación de voluntades y esfuerzos. Biomédica. 2006;26(Supl.1):1-3.

11. dos Reis DC, Gazzinelli A, Silva CA, Gazzinelli MF. Educação em saúde e representações sociais: uma experiencia no controle da leishmaniose tegumentar em area endêmica de Minas Gerais, Brasil. Cad Saude Publica. 2006;22:2301-10.

12. Santos JB, Lauand L, Souza GS, Macedo VO. Fatores sócio-económicos e atitudes em relaçao à prevenção domiciliar da leishmaniose tegumentar americana, em uma área endêmica do sul da Bahia, Brazil. Cad Saude Publica. 2000;16:701-8.

13. Moreira R da C, Rebelo JM, Gama ME, Costa JM. Nivel de conhecimentos sobre leishmaniose tegumentar americana (LTA) e uso de terapias alternativas por populações de uma área endêmica da Amazônia do Maranhão, Brasil. Cad Saude Publica. 2002;18:187-95.

14. Vásquez ML, Kroeger A, Lipowsky R, Alzate A. Conceptos populares sobre la leishmaniasis cutánea en Colombia y su aplicabilidad en programas de control. Bol Of Sanit Panam. 1991;110:402-12.

15. Pardo R, Carvajal A, Ferro C, Davies C. Effect of knowledge and economic status on sandfly control activities by householders at risk of cutaneous leishmaniasis in the subandean region of Huila department, Colombia. Biomédica. 2006;26(Supl.1): 167-79.

16. López-Vélez R, Molina-Moreno R. Cambio climático en España y riesgo de enfermedades infecciosas y parasitarias transmitidas por artrópodos y roedores. Rev Esp Salud Pública. 2005;79:177-90.

17. Dujardin JC. Risk factors in the spread of leishmaniasis: towards integrated monitoring? Trends Parasitol. 2006;22:4-6.

18. Herwaldt BL. Leishmaniasis. Lancet. 1999;354:1191-9.

19. Scorza JV, Valera M, Moreno E, Jaimes R. Epidemiologic survey of cutaneous leishmaniasis: an experience in Mérida, Venezuela. Bull Pan Am Health Organ. 1983;17:361-74.

20. Scorza JV. Información ecológica sobre Phlebotominae de Venezuela. Bol Dir Malariol San Amb. 1989;29:1-9.

21. García B. Aporte de la etnografía en el conocimiento de los códigos socioculturales de la leishmaniasis cutánea localizada en un programa de educación para la salud, en Venezuela. Cad Saude Publica. 2007;(Supl.1):S75-83.

22. Arias J, Beltrán F, Desjeux P, Walton B. Epidemiología y control de la leishmaniasis en las Américas, por país o territorio. Cuaderno técnico No. 44. Washington, D.C.: Organización Panamericana de la Salud; 1996.

23. Suárez YE, Fabré Y, Soca M, Fuentes M, Cabrera C, Álvarez J. Metodología para el análisis de algunos indicadores de riesgo asociados al manejo territorial de las zoonosis. Rev Electro Vet. 2006;7:1-8.

24. Young DG, Duncan MA. Guide to the identification and geographic distribution of Lutzomyia sand flies in Mexico, West Indies, Central and South America (Diptera: Psychodidea). Mem Ann Ent Inst. 1994;54:50881.

25. Dobles-Ulloa A, Perriard C. Representations, attitudes, and practices related to cutaneous leishmaniasis in people from Acosta country, San José province, Costa Rica. An exploratory anthropological study. Cad Saude Publica. 1994;10:181-9.

26. Weigel MM, Armijos RX, Racines RJ, Zurita C, Izurieta R, Herrera E, et al. Cutaneous leishmaniasis in subtropical Ecuador: popular perceptions, knowledge, 
and treatment. Bull Pan Am Health Organ. 1994;28: 142-55.

27. Isaza DM, Restrepo BN, Arboleda M, Casas E, Hinestroza H, Yurgaqui T. La leishmaniasis: conocimientos y prácticas en poblaciones de la costa del Pacífico de Colombia. Rev Panam Salud Pública. 1999;6:177-84

28. Yadon Z, Rodrigues L, Davies C, Quigley M. Indoor and peridomestic transmission of American cutaneous leishmaniasis in northwestern Argentina: A retrospective case-control study. Am J Trop. Med Hyg. 2003;68:519-26.

29. Desjeux $\mathbf{P}$. The increase in risk factors for leishmaniasis worldwide. Trans R Soc Trop Med Hyg. 2001;95:239-43.

30. Ampuero J, Urdaneta M, Macedo V de O. Risk factors for cutaneous leishmaniasis transmission in children aged 0 to 5 years in an endemic area of Leishmania (Viannia) braziliensis. Cad Saude Publica. 2005;21:16170.

31. Campbell-Lendrum D, Dujardin JP, Martinez E, Feliciangeli MD, Perez JE, Silans LN, et al. Domestic and peridomestic transmission of American cutaneous leishmaniasis: changing epidemiological patterns present new control opportunities. Mem Inst Oswaldo Cruz. 2001;96:159-62.

32. Salomón OD, Sosa S, Canini L, Córdoba E. Tegumentary leihsmaniasis in an area with epidemic levels of transmission, Salta, Argentina, 1988. Medicina. 2001;61:284-93.

33. Walsh JF, Molyneux DH, Birley MH. Deforestation: effects on vector-borne disease. Parasitology. 1993;106(Suppl):S55-75.

34. Gramiccia M, Gradoni L. The current status of zoonotic leishmaniases and approaches to disease control. Int J Parasitol. 2005;35:1169-80.

35. Kroeger A, Avila EV, Morrison L. Insecticide impregnated curtains to control domestic transmission of cutaneous leishmaniasis in Venezuela: cluster randomised trial. BMJ. 2002;325:810-3.

36. Travi BL, Jaramillo C, Montoya J, Segura I, Zea A, Goncalves A, et al. Didelphis marsupialis, an important reservoir of Trypanosoma (Schizotrypanum) cruzi and Leishmania (Leishmania) chagasi in Colombia. Am J Trop Med Hyg. 1994;50:557-65.
37. Alexander B, Barbosa EB, Haigh E, Almeida LL. Transmission of Leishmania in coffee plantations of Minas Gerais, Brazil. Mem Inst Oswaldo Cruz. 2002;97:627-30

38. Nieves E. Problemas de colonización de especies flebotominas bajo condiciones de laboratorio, con especial referencia a Lutzomyia youngi, Lutzomyia ovallesi y Lutzomyia migonei. Mérida: Facultad de Ciencias, Universidad de los Andes; 1995. p. 113.

39. Nieves E, Ribeiro A, Brazil R. Physical factors influencing the oviposition of Lutzomyia migonei (Diptera: Psychodidae) in laboratory conditions. Mem Inst Oswaldo Cruz. 1997;92:733-7.

40. Scorza JV, Castillo L, Rezzano S, Marquez M, Marquez JC. El papel del cafeto en la endemicidad de la leishmaniasis cutánea en Venezuela. Bol Malariol San Amb. 1985;25:82-8.

41. Traviezo LE. Phlebotomine sandflies in the southeast of Lara state, Venezuela. Biomédica. 2006;26(Suppl.1): 73-81.

42. Añez N, Cazorla D, Nieves E. Registro de especies flebotominas en focos endémicos para leishmaniasis en el estado Mérida, Venezuela. Bol Dir Malariol San Amb. 1989;29:12-34.

43. Salomon OD, Wilson ML, Munstermann LE, Travi BL. Spatial and temporal patterns of phlebotomine sand flies (Dipthera: Psychodidae) in cutaneous leishmaniasis focus in northern Argentina. J Med Entomol. 2004;41:33-9.

44. Azevedo AC, Rangel EF, Quieroz RG. Lutzomyia migonei (França, 1920) naturally infected with peripylarian flagellates in Baturité, a focus of leishmaniasis in Ceará State, Brazil. Mem Inst Oswaldo Cruz. 1990;85:479.

45. Nieves E, Pimienta PF. Development of Leishmania (Viannia) braziliensis and Leishmania (Leishmania) amazonensis in the sand fly Lutzomyia migonei (Diptera: Psychodidae). J Med Entomol. 2000;37:134-40.

46. Costa JM, Vale KC, França F, Costa MA, da Silva JO, Lago EL, et al. A leishmaniose tegumentar americana em uma região endêmica como fator de mobilização comunitária. Rev Soc Bras Med Trop. 1994;27:255-7. 